\title{
Nonsustained atrial tachycardia in 24-hour Holter monitoring: a potential cardiac source of embolism in acute ischemic stroke
}

\author{
Taedong Ok ${ }^{1}$, Seung Ha Lee ${ }^{1}$, Jong-Youn Kim ${ }^{2}$, Kyung-Yul Lee ${ }^{1}$, Yo Han Jung ${ }^{1}$ \\ ${ }^{1}$ Department of Neurology, Gangnam Severance Hospital, Yonsei University College of Medicine, Seoul, Korea; ${ }^{2}$ Cardiology Division, Gangnam \\ Severance Hospital, Yonsei University College of Medicine, Seoul, Korea \\ Contributions: (I) Conception and design: T Ok, KY Lee, YH Jung; (II) Administrative support: T Ok; (III) Provision of study materials or patients: \\ JY Kim; (IV) Collection and assembly of data: T Ok, SH Lee; (V) Data analysis and interpretation: T Ok; (VI) Manuscript writing: All authors; (VII) \\ Final approval of manuscript: All authors. \\ Correspondence to: Yo Han Jung, MD, PhD. Department of Neurology, Gangnam Severance Hospital, Yonsei University College of Medicine, 211, \\ Eonju-ro, Gangnam-gu, Seoul 06273, Korea. Email: yhjung@yuhs.ac.
}

Background: Whether nonsustained atrial tachycardia (NSAT) has a causative role similar to paroxysmal
atrial fibrillation (AF) in ischemic stroke is unclear. We investigated the clinical and imaging features of
ischemic stroke patients with NSAT to demonstrate that these patients would have a higher proportion of
embolic strokes.
Methods: We retrospectively reviewed ischemic stroke patients who underwent Holter monitoring and
selected patients with NSAT. The clinical and imaging characteristics were compared between patients
with and without NSAT, and the risk factors for embolic stroke were evaluated. Moreover, the images
of the selected patients were analyzed according to the Trials of Org 10172 in Acute Stroke Treatment
classification.

Results: From a total of 1,051 patients who had 24-hour Holter monitoring, 681 patients were selected for the study. Among the selected patients, NSAT was detected in 243 patients. The patients with NSAT had a significantly higher proportion of imaging findings suggestive of cerebral embolism compared with patients without NSAT $(27 \%$ vs. $14 \%, \mathrm{P}<0.001)$. Moreover, the presence of NSAT was a statistically significant factor associated with imaging findings suggestive of cerebral embolism in the univariate (OR, 2.22; 95\% CI, 1.51-3.27; $\mathrm{P}<0.001)$ and multivariate $(\mathrm{OR}, 2.26$; 95\% CI, 1.53-3.34; $\mathrm{P}<0.001)$ analyses. The patients with NSAT had a significantly older age at diagnosis, higher proportion of female sex, higher proportion of hypertension, lower proportion of smokers, higher $\mathrm{CHA}_{2} \mathrm{DS}_{2}$-VASc score, and higher left atrium index value compared with patients without NSAT.

Conclusions: The embolic pattern of acute ischemic stroke in patients with NSAT was frequently observed and shared clinical characteristics of AF rather than those of atherosclerosis. As NSAT may be a potential source of cardiac embolism, we suggest a more intensive search for modifiable risk factors such as $\mathrm{AF}$ in ischemic stroke in patients with NSAT.

Keywords: Holter monitoring; cryptogenic stroke; paroxysmal atrial fibrillation (paroxysmal AF)

Submitted Oct 04, 2021. Accepted for publication Jan 28, 2022.

doi: $10.21037 / \mathrm{atm}-21-5245$

View this article at: https://dx.doi.org/10.21037/atm-21-5245

\section{Introduction}

Cryptogenic stroke is defined by the Trial of Org 10172 in Acute Stroke Treatment (TOAST) classification as when the diagnostic assessment is incomplete, no cause for the stroke is found despite an extensive evaluation, or a cause for the stroke cannot be established because of multiple possible etiologies (1). Cryptogenic ischemic strokes account for approximately $25-33 \%$ of ischemic strokes, and paroxysmal 
atrial fibrillation (AF) is often suspected as the cause of cryptogenic stroke. The incidence of stroke in patients with paroxysmal AF is similar to that in patients with sustained $\mathrm{AF}$ (2). Therefore, identification of AF is essential because the risk of recurrent stroke can be significantly reduced by the use of anticoagulation in patients with ischemic stroke having AF.

A single electrocardiogram (EKG) recording or 24-hour Holter monitoring has a low diagnostic yield in detecting paroxysmal $\mathrm{AF}$, but detection of $\mathrm{AF}$ has improved with the use of ambulatory cardiac monitoring and implantable devices $(3,4)$. These devices have also enabled the detection of other aberrations such as atrial premature beats (APB), supraventricular ectopic activity, and nonsustained atrial tachycardia (NSAT) in patients with ischemic stroke. Although NSAT is often considered a benign phenomenon, it often precedes episodes of $\mathrm{AF}$ in patients with cryptogenic stroke (5-7). It remains unclear whether NSAT has a causative role similar to paroxysmal $\mathrm{AF}$ in ischemic stroke (8-10). In clinical practice, the presence of NSAT is not regarded as a cardiac source of embolism, and antiplatelets are generally prescribed for patients with ischemic stroke and NSAT for secondary prevention.

We hypothesized that patients with ischemic stroke and NSAT would have a higher proportion of embolic strokes than patients without NSAT. In this investigation, we studied the clinical characteristic and imaging findings of patients with NSAT and evaluated the risk factors associated with imaging findings suggestive of cerebral embolism. In addition, we investigated the clinical and laboratory findings of patients with stroke and NSAT on 24-hour Holter monitoring and compared them between patients with and without an embolic pattern of infarction on brain images. We present the following article in accordance with the STROBE reporting checklist (available at https://atm. amegroups.com/article/view/10.21037/atm-21-5245/rc).

\section{Methods}

The study was conducted in accordance with the Declaration of Helsinki (as revised in 2013). The institutional review board of Severance Hospital, Yonsei University Health System, approved this retrospective study (3-2014-0264). Written informed consent for participation was waived for this retrospective study. We retrospectively reviewed 1,752 patients with acute ischemic stroke who were admitted to the neurology department of a tertiary university hospital between January 2012 and May 2018.
From 1,051 patients who had 24-hour Holter monitoring, we initially selected 985 patients who underwent (I) brain magnetic resonance imaging, and either magnetic resonance angiography or computed tomography angiography; and (II) transthoracic echocardiography. We excluded 304 patients with any potential cardiac sources of embolism, including (I) a history of AF; (II) documented AF on EKG, 24hour Holter monitoring, or echocardiography; (III) patent foramen ovale; and (IV) aortic atheroma with thickness $\geq 4 \mathrm{~mm}$. Baseline characteristics, underlying vascular risk factors (hypertension, diabetes mellitus, coronary artery disease, dyslipidemia, and current smoking), prior history of transient ischemic attack or ischemic stroke, stroke severity as per the National Institutes of Health Stroke Scale (NIHSS), cardiac parameters, and etiology subtype based on TOAST classification were collected from 681 selected patients. The radiographic images were also carefully reviewed to find images suggestive of cerebral embolism. Imaging findings suggestive of cerebral embolism were defined when any of the following features were found: (I) simultaneous acute ischemic lesions among multiple arterial territories (Figure 1), (II) presence of an isolated acute cortical lesion, (III) angiographic evidence of isolated arterial occlusion or recanalization without underlying arterial stenosis on follow-up angiography (Figure 2) (11). The imaging findings were investigated by a neurology specialist with five years of experience who was blinded to the clinical results.

The selected patients were divided into two groups according to the presence or absence of documented NSAT on 24-hour Holter monitoring. NSAT was defined as three or more consecutive APB with a rate of $>100$ beats/min and lasting <30 s. We defined APB when the following criteria were met: (I) a reduced RR interval $\geq 25 \%$, (II) the presence of a P wave, and (III) a QRS width $<0.12 \mathrm{~s}$ (12). The incidence of imaging findings suggestive of cerebral embolism was compared between the two groups. We analyzed the risk factors related to imaging findings suggestive of cerebral embolism.

Patients with NSAT were further divided into two groups according to the presence or absence of image findings suggestive of cerebral embolism. To find characteristic features in patients with image findings suggestive of cerebral embolism, the vascular risk factors, cardiac parameters, specific parameters related to NSAT, and lipid profiles were compared.

Statistical analysis was performed using IBM SPSS Statistics 25.0. Continuous variables were presented 

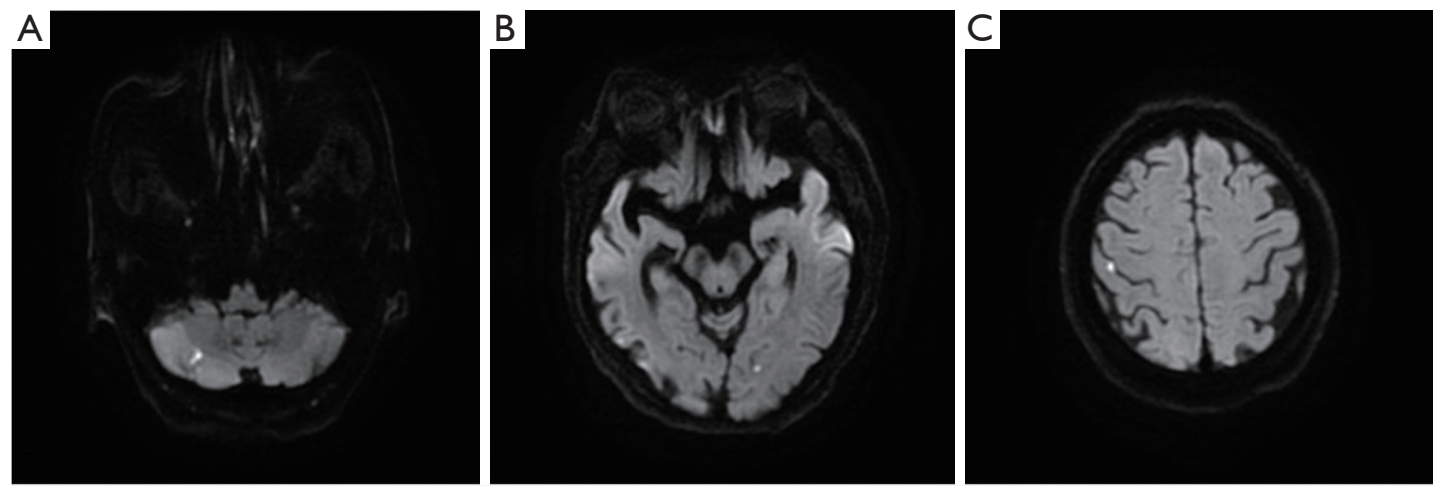

Figure 1 Example of simultaneous acute ischemic lesions in multiple arterial territories on the diffusion-weighted image. Concurrent acute ischemic lesions detected at the (A) right posterior inferior cerebellar artery territory, (B) left posterior cerebral artery territory, and (C) right middle cerebral artery territory.
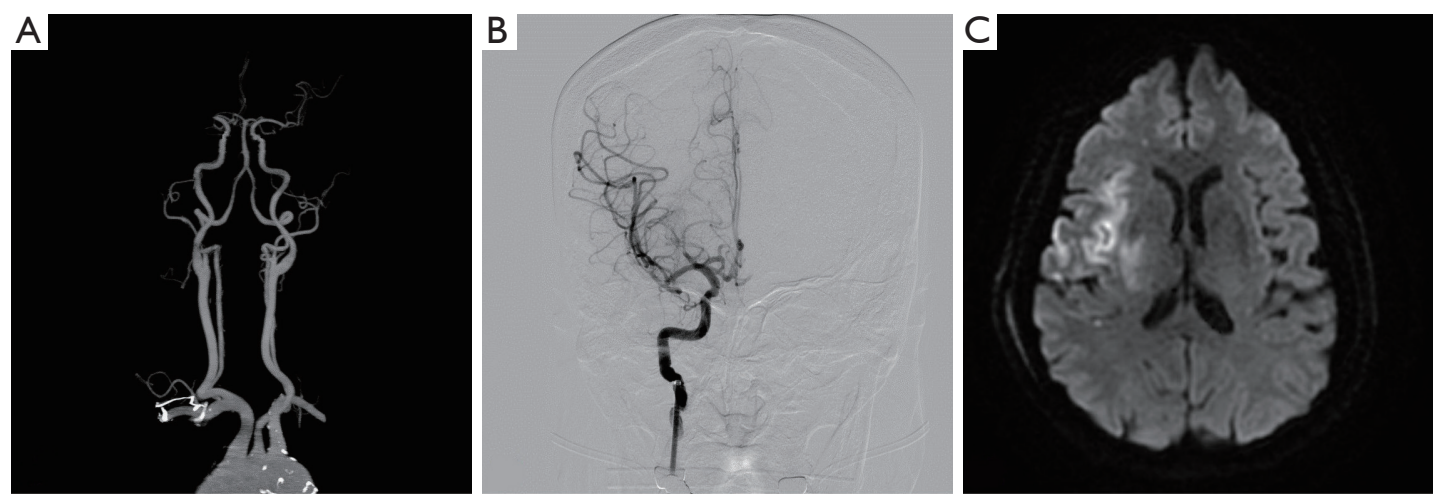

Figure 2 Example of angiographic evidence of cut-off sign and recanalization. (A) Cut-off sign at the right middle cerebral artery without evidence of atherosclerosis on CT angiography. (B) Cerebral angiography after recanalization by mechanical thrombectomy. (C) Diffusionweighted image one day after recanalization.

as means with standard deviations or as medians with interquartile ranges and were compared using the independent two-sample $t$-test or Mann-Whitney $\mathrm{U}$ test, respectively. Categorical variables were presented as counts (percentage) and were compared using the chisquare test or Fisher's exact test. Risk factors for image findings suggestive of cerebral embolism were evaluated using a logistic regression model. Univariable analyses were performed with variables, including the presence of NSAT, age at diagnosis, sex, vascular risk factors, and cardiac parameters. Multivariable analyses using variables with $\mathrm{P}$ values $<0.10$ were performed to identify the risk factors for image findings suggestive of cerebral embolism. A twosided $\mathrm{P}$ value $<0.05$ was considered statistically significant.

\section{Results}

The patient characteristics are summarized in Table 1. We identified 681 patients satisfying the inclusion and exclusion criteria of the study. The mean age was 63 years and 240 (35\%) of the patients were female. Four hundred (59\%) had hypertension, 208 (31\%) had diabetes mellitus, 54 (8\%) had coronary artery diseases, and 351 (52\%) had dyslipidemia. Seventy-seven $(11 \%)$ patients had a previous history of transient ischemic attack or ischemic stroke. The median initial NIHSS score was two.

Among the 681 patients, 243 (36\%) had documented NSAT on 24-hour Holter monitoring. The patients with NSAT had a significantly older age at diagnosis, 
Table 1 Clinical characteristics and imaging findings between patients with and without nonsustained atrial tachycardia in acute ischemic stroke patients

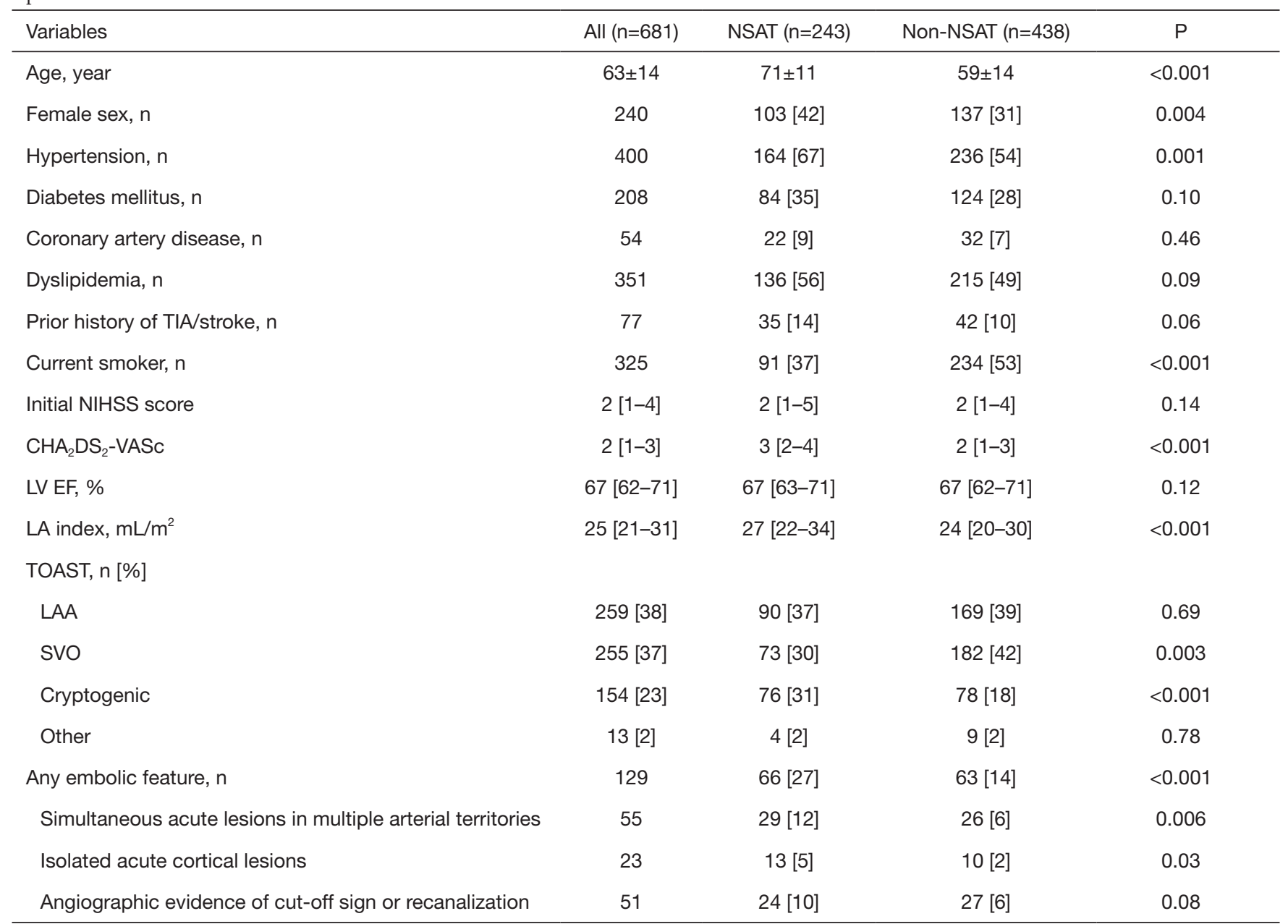

NSAT, nonsustained atrial tachycardia; TIA, transient ischemic attack; NIHSS, National Institutes of Health Stroke Scale; $\mathrm{CHA}_{2} \mathrm{DS} \mathrm{S}_{2}-\mathrm{VASc}$ congestive heart failure, hypertension, age $\geq 75$ years, diabetes, prior stroke or TIA, vascular disease, age 65-74 years, sex; LV EF, left ventricular ejection fraction; LA, left atrium; TOAST, Trial of Org 10172 in Acute Stroke Treatment; LAA, large artery atherosclerosis; SVO, small vessel occlusion; cryptogenic, undetermined-more than two causes or undetermined-negative; other, other determined etiologies.

and a higher proportion was female compared to those without NSAT. The patients with NSAT also had a higher proportion with hypertension than those without NSAT. In patients with NSAT, the $\mathrm{CHA}_{2} \mathrm{DS}_{2}$-VASc scores and left atrium (LA) index values were higher. Moreover, a lower proportion of NSAT patients smoked compared to those without NSAT. There was no significant difference in the proportion of diabetes mellitus, coronary artery disease, dyslipidemia, prior history of transient ischemic attack or ischemic stroke, NIHSS score, and left ventricular ejection fraction. Upon TOAST classification, patients with NSAT had more classified as cryptogenic, and those without NSAT had more classified as small vessel occlusion. The incidence of imaging findings suggestive of cerebral embolism was also compared between the two groups. The patients with NSAT had a significantly higher proportion of imaging findings suggestive of cerebral embolism than those without NSAT (27\% vs. $14 \%, \mathrm{P}<0.001)$ (Table 1).

We performed a logistic regression analysis for imaging findings suggestive of cerebral embolism. Among the risk factors, the presence of the NSAT was a statistically significant factor associated with imaging findings suggestive of cerebral embolism in univariate [odds ratio (OR), 2.22; 95\% CI, 1.51-3.27; $\mathrm{P}<0.001]$ and multivariate $(\mathrm{OR}, 2.26$; 
Table 2 Results of logistic regression analysis for images suggestive of cerebral embolism in patients with acute ischemic stroke

\begin{tabular}{|c|c|c|c|c|}
\hline Variables & \multicolumn{2}{|c|}{ Univariable model } & \multicolumn{2}{|c|}{ Multivariable model } \\
\hline NSAT & $2.22(1.51-3.27)$ & $<0.001$ & $2.26(1.53-3.34)$ & $<0.001$ \\
\hline Age, year & $1.01(1.00-1.02)$ & 0.16 & - & - \\
\hline Female sex & $1.11(0.75-1.65)$ & 0.60 & - & - \\
\hline Diabetes mellitus & $0.82(0.53-1.25)$ & 0.35 & - & - \\
\hline Coronary artery disease & $0.41(0.16-1.06)$ & 0.07 & $0.39(0.15-1.00)$ & 0.0504 \\
\hline Dyslipidemia & $0.81(0.55-1.19)$ & 0.28 & - & - \\
\hline Prior history of TIA/stroke & $1.04(0.57-1.89)$ & 0.90 & - & - \\
\hline LV EF, \% & $1.01(0.98-1.04)$ & 0.51 & - & - \\
\hline $\mathrm{LA}$ index, $\mathrm{mL} / \mathrm{m}^{2}$ & $1.00(0.98-1.03)$ & 0.76 & - & - \\
\hline
\end{tabular}

OR, odds ratio; $\mathrm{Cl}$, confidence interval; NSAT, nonsustained atrial tachycardia; TIA, transient ischemic attack; NIHSS, National Institutes of Health Stroke Scale; $\mathrm{CHA}_{2} \mathrm{DS}_{2}$-VASc, congestive heart failure, hypertension, age $\geq 75$ years, diabetes, prior stroke or TIA, vascular disease, age 65-74 years, sex; LV EF, left ventricular ejection fraction; LA, left atrium.

95\% CI, 1.53-3.34; $\mathrm{P}<0.001$ ) analyses (Table 2). The history of coronary artery disease was associated with imaging findings suggestive of cerebral embolism; however, it was not statistically significant in the univariable and multivariable analysis (OR, 0.39; 95\% CI, 0.15-1.00; $\mathrm{P}=0.05)$. None of the other variables, including age, sex, other vascular risk factors, prior history of transient ischemic attack or ischemic stroke, $\mathrm{CHA}_{2} \mathrm{DS}_{2}$-VASc score, left ventricular ejection fraction, and LA index value were associated with imaging findings suggestive of cerebral embolism. Further analysis of imaging features by TOAST classification revealed that in patients classified as large artery atherosclerosis (LAA), patients with NSAT showed a significantly higher proportion of angiographic evidence of cut-off or recanalization than patients without NSAT (Table S1).

Patients with NSAT were divided into two groups based on imaging findings suggestive of cerebral embolism: 66 and 177 patients with and without embolic features, respectively. The comparison of clinical characteristics and laboratory findings between the two groups is shown in Table S2. Patients with embolic features had a significantly higher initial NIHSS score $\{3$ [interquartile range (IQR), 1-9] $v$ s. 2 (IQR, 1-4), $\mathrm{P}=0.002\}$ compared with patients without embolic features. The total number of APB, number of
$\mathrm{APB}$ in the longest run, and LA index showed no significant difference between the two groups.

\section{Discussion}

A major finding of this study is that patients with acute ischemic stroke and NSAT had a higher proportion of embolic imaging patterns than those without NSAT. Moreover, NSAT was independently associated with such imaging findings. A previous study has shown that the cardioembolic stroke subtype tends to occur in older patients (age $>70$ years), a higher proportion of females, and demonstrates more severe stroke symptoms (mean NIHSS of 8 compared to 5 of LAA). In contrast, the LAA stroke subtype tends to occur in a higher number of patients with hypercholesterolemia and a higher proportion of smokers (13). In our study, the patients with NSAT were older, a higher proportion was female, a higher proportion had a history of hypertension, and they had higher $\mathrm{CHA}_{2} \mathrm{DS}_{2}$-VASc scores and LA index values, which are factors known to be associated with AF $(14,15)$. Similarly, patients without NSAT included a higher proportion of smokers, which is associated with atherosclerosis. Moreover, the logistic regression model showed that NSAT 
was positively associated with embolic features, while a history of coronary artery disease was negatively associated with NSAT, although this was not statistically significant. Patients with acute ischemic stroke and NSAT were likely to share the risk factors for AF rather than those for atherosclerosis.

About one-third of ischemic strokes are classified as cryptogenic strokes, and covert $\mathrm{AF}$ is often suspected as the cause. Since patients with a definite cardioembolic source such as $\mathrm{AF}$ were excluded from our study population, we could find a higher proportion of embolic stroke in patients with NSAT. Furthermore, patients with NSAT were classified as cryptogenic more often and as small vessel occlusion less often according to the TOAST classification compared with those without NSAT. Additionally, although there was no difference in the proportion of patients classified as LAA between patients with and without NSAT, analysis of radiographic images showed that the patients with NSAT had significantly more isolated arterial cutoff signs or recanalization without atherosclerosis in other vessels. This could mean that LAA with isolated cut-off and recanalization without any residual stenosis could be caused by cardiac embolism rather than atherosclerosis, despite being classified as LAA according to the TOAST classification. These features suggest that a significant proportion of patients with acute ischemic stroke and NSAT might have a cardiogenic mechanism though they were classified as having LAA or cryptogenic stroke.

The mechanism of embolic stroke in patients with NSAT is unclear. Frequent APB represents a proarrhythmic tendency and could be the precursor or the first manifestation of subclinical AF (16-19). The number of $\mathrm{APB}$ on a routine 24-hour Holter EKG was shown to be a strong predictor of subclinical $\mathrm{AF}$ in a subgroup analysis of the EMBRACE trial (5). In a study with a large and demographically diverse sample of patients, paroxysmal supraventricular tachycardia was associated with ischemic stroke and may account for the rationale behind some proportion of cryptogenic stroke (20). Moreover, extendedduration monitoring or long-term EKG monitoring tools such as inpatient cardiac telemetry or implantable loop recorders have increased the detection of AF in cryptogenic stroke patients (21). Our study showed that patients with NSAT had higher $\mathrm{CHA}_{2} \mathrm{DS}_{2}$-VASc scores and LA index values with a higher proportion of patients with cryptogenic stroke. Subgroup analysis with NSAT patients with and without an embolic feature showed no significant differences in the total APB or longest run of APB between the two groups. Therefore, NSAT may be a marker of covert AF not detected in a particular Holter monitoring rather than being a direct cause of clot formation. Further study using extended cardiac monitoring to detect AF in patients with NSAT is necessary to prove the role of NSAT in ischemic stroke.

Our study has several limitations. First, there may be selection bias because not all the acute ischemic patients underwent 24-hour Holter monitoring due to various reasons such as cost and low probability of a cardiogenic cause, and we obtained data from a single center. However, because we included numerous consecutive patients, we expect the selection bias to be diluted. Second, some patients with definite cardioembolic causes might be included in the study since not all the patients underwent transesophageal echocardiography. However, we expect that number of patients to be small because most patients with a suspicion of cardioembolic stroke routinely undergo transesophageal echocardiography in our clinical setting. Third, our study was retrospective in nature. Although prospective population-based studies have not been conducted, we believe our study might provide preliminary insights into the role of NSAT in patients with ischemic stroke.

\section{Conclusions}

In conclusion, NSAT was significantly associated with embolic patterns in acute ischemic stroke patients. Moreover, NSAT was an independent risk factor for embolic stroke. The patients with NSAT shared the risk factors of AF rather than those of atherosclerosis. This could mean that NSAT could be a predisposing event of AF. Prolonged EKG monitoring could potentially offer more opportunities to detect AF and, eventually, a chance to identify modifiable risk factors for recurrent stroke.

\section{Acknowledgments}

We would like to thank Editage (www.editage.co.kr) for English language editing. This work was presented as an E-poster at the 5th European Stroke Organization Conference (ESOC 2019) (AS15-048).

Funding: None.

\section{Footnote}

Reporting Checklist: The authors have completed the 
STROBE reporting checklist. Available at https://atm. amegroups.com/article/view/10.21037/atm-21-5245/rc

Data Sharing Statement: Available at https://atm.amegroups. com/article/view/10.21037/atm-21-5245/dss

Peer Review File: Available at https://atm.amegroups.com/ article/view/10.21037/atm-21-5245/prf

Conflicts of Interest: All authors have completed the ICMJE uniform disclosure form (available at https://atm. amegroups.com/article/view/10.21037/atm-21-5245/coif). The authors have no conflicts of interest to declare.

Ethical Statement: The authors are accountable for all aspects of the work in ensuring that questions related to the accuracy or integrity of any part of the work are appropriately investigated and resolved. The study was conducted in accordance with the Declaration of Helsinki (as revised in 2013). Ethical approval was granted by the institutional review board of Severance Hospital, Yonsei University Health System (approval number: 3-2014-0264). Written informed consent for participation was waived for this retrospective study.

Open Access Statement: This is an Open Access article distributed in accordance with the Creative Commons Attribution-NonCommercial-NoDerivs 4.0 International License (CC BY-NC-ND 4.0), which permits the noncommercial replication and distribution of the article with the strict proviso that no changes or edits are made and the original work is properly cited (including links to both the formal publication through the relevant DOI and the license). See: https://creativecommons.org/licenses/by-nc-nd/4.0/.

\section{References}

1. Nouh A, Hussain M, Mehta T, et al. Embolic Strokes of Unknown Source and Cryptogenic Stroke: Implications in Clinical Practice. Front Neurol 2016;7:37.

2. Hart RG, Pearce LA, Rothbart RM, et al. Stroke with intermittent atrial fibrillation: incidence and predictors during aspirin therapy. Stroke Prevention in Atrial Fibrillation Investigators. J Am Coll Cardiol 2000;35:183-7.

3. Gladstone DJ, Spring M, Dorian P, et al. Atrial fibrillation in patients with cryptogenic stroke. $\mathrm{N}$ Engl J Med 2014;370:2467-77.
4. Sanna T, Diener HC, Passman RS, et al. Cryptogenic stroke and underlying atrial fibrillation. $\mathrm{N}$ Engl J Med 2014;370:2478-86.

5. Gladstone DJ, Dorian P, Spring M, et al. Atrial Premature Beats Predict Atrial Fibrillation in Cryptogenic Stroke. Stroke 2015;46:936-41.

6. Healey JS, Connolly SJ, Gold MR, et al. Subclinical atrial fibrillation and the risk of stroke. $\mathrm{N}$ Engl J Med 2012;366:120-9.

7. Tayal AH, Tian M, Kelly KM, et al. Atrial fibrillation detected by mobile cardiac outpatient telemetry in cryptogenic TIA or stroke. Neurology 2008;71:1696-701.

8. Binici Z, Intzilakis T, Nielsen OW, et al. Excessive supraventricular ectopic activity and increased risk of atrial fibrillation and stroke. Circulation 2010;121:1904-11.

9. Larsen BS, Kumarathurai P, Falkenberg J, et al. Excessive Atrial Ectopy and Short Atrial Runs Increase the Risk of Stroke Beyond Incident Atrial Fibrillation. J Am Coll Cardiol 2015;66:232-41.

10. Swiryn S, Orlov MV, Benditt DG, et al. Clinical Implications of Brief Device-Detected Atrial Tachyarrhythmias in a Cardiac Rhythm Management Device Population: Results from the Registry of Atrial Tachycardia and Atrial Fibrillation Episodes. Circulation 2016;134:1130-40.

11. Arsava EM, Bas DF, Atalar E, et al. Ischemic stroke phenotype in patients with nonsustained atrial fibrillation. Stroke 2015;46:634-40.

12. Chung H, Joung B, Lee KY, et al. Left Atrial Volume Index Predicts Recurrence of Stroke in Patients with Nonsustained Atrial Tachycardia. J Stroke Cerebrovasc Dis 2015;24:2408-15.

13. Grau AJ, Weimar C, Buggle F, et al. Risk factors, outcome, and treatment in subtypes of ischemic stroke: the German stroke data bank. Stroke 2001;32:2559-66.

14. Lip GY, Nieuwlaat R, Pisters R, et al. Refining clinical risk stratification for predicting stroke and thromboembolism in atrial fibrillation using a novel risk factor-based approach: the euro heart survey on atrial fibrillation. Chest 2010;137:263-72.

15. Seko Y, Kato T, Haruna T, et al. Association between atrial fibrillation, atrial enlargement, and left ventricular geometric remodeling. Sci Rep 2018;8:6366.

16. Chong BH, Pong V, Lam KF, et al. Frequent premature atrial complexes predict new occurrence of atrial fibrillation and adverse cardiovascular events. Europace 2012;14:942-7.

17. John RM, Stevenson WG. Predicting atrial fibrillation: 
can we shape the future? Eur Heart J 2015;36:145-7.

18. Kramer DB, Zimetbaum PJ. Ectopy and expectations: can we predict atrial fibrillation, and should we try? Ann Intern Med 2013;159:787-8.

19. Murakoshi N, Xu D, Sairenchi T, et al. Prognostic impact of supraventricular premature complexes in communitybased health checkups: the Ibaraki Prefectural Health Study. Eur Heart J 2015;36:170-8.

20. Kamel H, Elkind MS, Bhave PD, et al. Paroxysmal

Cite this article as: Ok T, Lee SH, Kim JY, Lee KY, Jung YH. Nonsustained atrial tachycardia in 24-hour Holter monitoring: a potential cardiac source of embolism in acute ischemic stroke. Ann Transl Med 2022;10(8):433. doi: 10.21037/atm-21-5245 supraventricular tachycardia and the risk of ischemic stroke. Stroke 2013;44:1550-4.

21. Kułach A, Dewerenda M, Majewski M, et al. Supraventricular Runs in 7-Day Holter Monitoring Are Related to Increased Incidence of Atrial Fibrillation in a 3-Year Follow-Up of Cryptogenic Stroke Patients Free from Arrhythmia in a 24 h-Holter. J Cardiovasc Dev Dis 2021;8:81. 


\section{Supplementary}

Table S1 Comparison of imaging features between patients with and without nonsustained atrial tachycardia by TOAST classification (LAA and cryptogenic)

\begin{tabular}{|c|c|c|c|c|c|c|}
\hline Variables & \multicolumn{3}{|c|}{ LAA $(n=259)$} & \multicolumn{3}{|c|}{ Cryptogenic $(n=154)$} \\
\hline Any embolic feature & 21 [23] & $23[13]$ & 0.047 & $44[58]$ & 38 [49] & 0.25 \\
\hline $\begin{array}{l}\text { Simultaneous acute lesions in multiple arterial } \\
\text { territories }\end{array}$ & $0[0]$ & $1[1]$ & $>0.99$ & 29 [38] & 23 [29] & 0.26 \\
\hline Isolated acute cortical lesions & $0[0]$ & $0[0]$ & - & $13[17]$ & 10 [13] & 0.46 \\
\hline
\end{tabular}

${ }^{\dagger}$, Chi square test or Fisher's exact test were used. LAA, large artery atherosclerosis; cryptogenic, undetermined-more than two causes or undetermined-negative; other, other determined etiologies; NSAT, nonsustained atrial tachycardia.

Table S2 Clinical characteristics and laboratory findings between patients with and without imaging findings suggestive of cerebral embolism in acute ischemic stroke patients with nonsustained atrial tachycardia

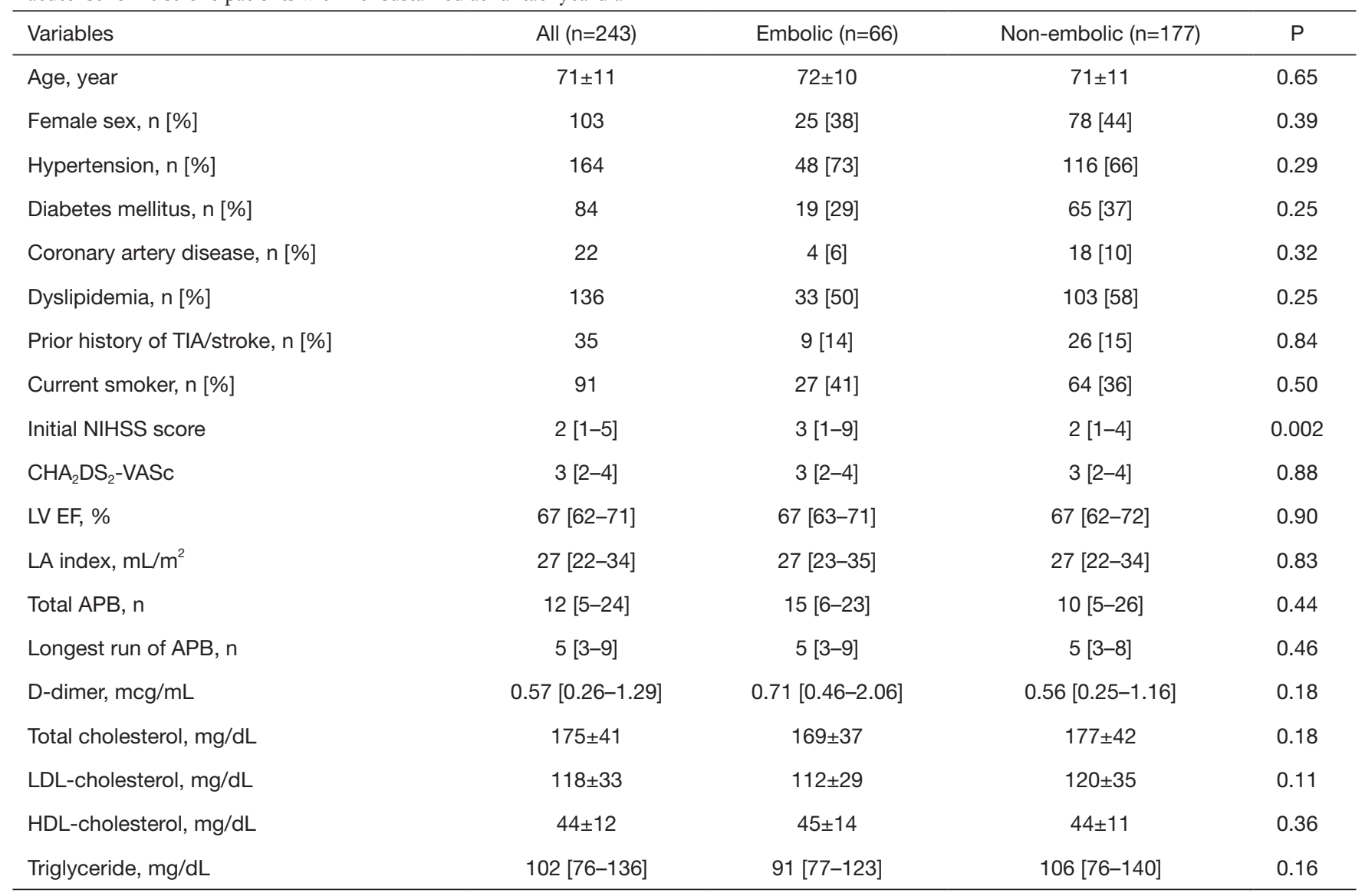

TIA, transient ischemic attack; NIHSS, National Institutes of Health Stroke Scale; $\mathrm{CHA}_{2} \mathrm{DS}_{2}-\mathrm{VASc}$, congestive heart failure, hypertension, age $\geq 75$ years, diabetes, prior stroke or TIA, vascular disease, age 65-74 years, sex; LV EF, left ventricular ejection fraction; LA, left atrium; APB, atrial premature beat; LDL, low-density lipoprotein; HDL, high-density lipoprotein. 